\title{
SPECTRAL THEORY FOR BOUNDARY VALUE PROBLEMS FOR ELLIPTIC SYSTEMS OF MIXED ORDER
}

\author{
BY GIUSEPPE GEYMONAT AND GERD GRUBB \\ Communicated by Robert Seeley, May 28, 1974
}

Introduction. For a closed, densely defined linear operator $T$ in a Hilbert space $H$, we define the essential spectrum ess sp $T$ as the complement in $\mathbf{C}$ of the set of $\lambda$ for which $T-\lambda$ is a Fredholm operator (with possibly nonzero index). Recall (cf. Wolf [7]) that $\lambda \in$ ess sp $T$ if and only if either $T-\lambda$ or $T^{*}-\bar{\lambda}$ has a singular sequence, i.e. a sequence $u_{k} \in H$ with $\left\|u_{k}\right\|=1$ for all $k,(T-\lambda) u_{k} \rightarrow 0$ (or $\left(T^{*}-\bar{\lambda}\right) u_{k} \rightarrow 0$ ) in $H$, but $u_{k}$ having no convergent subsequence in $H$. ess sp $T$ is closed and invariant under compact perturbations of $T$, and contains the accumulation points of the eigenvalue spectrum.

Let $\bar{\Omega}$ be an $n$-dimensional compact $C^{\infty}$ manifold with boundary $\Gamma$ and interior $\Omega=\bar{\Omega} \backslash \Gamma$. It is well known that when $A$ is a properly elliptic operator on $\bar{\Omega}$ of order $r>0$, the $L^{2}$-realization $A_{B}: u \mapsto A u$ with domain $D\left(A_{B}\right)=\left\{u \in L^{2}(\Omega)\left|A u \in L^{2}(\Omega), B u\right|_{\Gamma}=0\right\}$, defined by a boundary operator $B$ that covers $A$ (i.e. $\{A, B\}$ defines an elliptic boundary value problem), has ess $\operatorname{sp} A_{\mathrm{B}}=\varnothing$.

However, when $A$ is a system of mixed order, elliptic in the sense of Douglis and Nirenberg (cf. [1]), ess sp $A_{B}$ can be nonempty even when $\{A, B\}$ is elliptic with smooth coefficients and $\bar{\Omega}$ is compact. We study this phenomenon for a class of Douglis-Nirenberg systems of nonnegative order, determine the essential spectrum, and find the asymptotic behavior of the discrete spectrum at $+\infty$ for the selfadjoint lower bounded realizations.

Examples of the systems we consider are: The linearized Navier-Stokes operator and certain systems stemming from nuclear reactor analysis. A preliminary, less advanced account of the theory was given in [5].

\section{Preliminaries.}

1.1. For $q$ integer $>1$ there is given a set of integers $m_{1} \geqslant m_{2} \geqslant$

AMS (MOS) subject classifications (1970). Primary 35J45, 47B30, 35P20. 
$\cdots \geqslant m_{q^{\prime}}>m_{q^{\prime}+1}=\cdots=m_{q}=0$; we assume $1 \leqslant q^{\prime}<q$ and denote $\max m_{s}=m$. Let $A=\left(A_{s t}\right)_{s, t=1, \cdots, q}$ be a $q \times q$-matrix of differential operators of orders $m_{s}+m_{t}$ on $\overline{\bar{\Omega}} . \AA$ [1]), i.e. the principal symbol $\sigma^{0}(A)=\left(\sigma_{m_{s}+m_{t}}\left(A_{s t}\right)\right)$ has nonzero determinants on $T^{*}(\bar{\Omega}) \backslash 0$. It is useful to single out the zero order part of $A$ by splitting the rows and columns into the first $q^{\prime}$ and the last $q-q^{\prime}$ entries:

$$
\mathrm{A}=\left(\begin{array}{ll}
P & Q \\
R & M
\end{array}\right) ;
$$

here $P, Q$ and $R$ are of positive order, and $M$ is a multiplication operator.

1.2. The notation for boundary conditions follows Grubb $[4$, Chapter 3]: For $u=\left\{u_{1}, \cdots, u_{q}\right\}, \beta^{0} u$ denotes the Dirichlet data (the collection of the normal derivatives of each $u_{t}$ up to order $m_{t}-1$ ), and $\beta^{1} u$ denotes the remaining normal derivatives up to orders $m_{t}+m-1$, arranged as in [4]; $\beta u=\left\{\beta^{0} u, \beta^{1} u\right\}$ constitute the "Cauchy data". We consider boundary conditions of the form

$$
B^{00} \beta^{0} u=0, \quad B^{10} \beta^{0} u+B^{1} \widetilde{\mathbb{Q}}^{01} \beta^{1} u=0,
$$

where $\widetilde{\mathfrak{Q}}^{01}$ is a certain fixed surjective differential operator entering in Green's formula, and the $B^{*}$ are systems of differential operators of suitable orders (cf. [4], selfadjoint or semibounded realizations necessarily stem from boundary conditions of this form, and it can be shown that boundary problems in general reduce to at least inhomogeneous conditions on $\beta^{0} u$ and $\widetilde{\mathbb{Q}}^{01} \beta^{1} u$, the "reduced Cauchy data"). We assume thoughout that (1.2) covers A (satisfies the conditions in [1]).

2. The case of a manifold without boundary.

2.1. Assume first that $\bar{\Omega}$ is compact with $\Gamma=\varnothing$, i.e. $\bar{\Omega}=\Omega$. Then A has a parametrix $\widetilde{A}$, which we split in the same blocks as (1.1):

$$
\widetilde{A}=\left(\begin{array}{ll}
\widetilde{P} & \widetilde{Q} \\
\widetilde{R} & \widetilde{S}
\end{array}\right) ;
$$

here $\widetilde{P}, \widetilde{Q}$ and $\widetilde{R}$ are pseudodifferential operators of negative (mixed) order, and $\widetilde{S}$ is a ps.d.o. of order zero. A has only one $L^{2}$-realization, which we call $A$, 


$$
D(A)=\left\{u \in L^{2}(\Omega)^{q} \mid A u \in L^{2}(\Omega)^{q}\right\} .
$$

One finds by use of $\widetilde{A}$ that $D(A) \subset \prod_{t=1}^{q} H^{m} t(\Omega)$.

THEOREM 2.1. ess sp $A=\left\{\lambda \neq 0 \mid \lambda^{-1} \in \operatorname{ess} \operatorname{sp} \widetilde{S}\right\}=\left\{\lambda \neq 0 \mid \lambda^{-1}\right.$ is an eigenvalue for $\sigma^{0}(\widetilde{S})(x, \xi)$ for some $\left.(x, \xi) \in T^{*}(\bar{\Omega}) \backslash 0\right\}=\{\lambda \mid A-\lambda$ is not elliptic\}.

SKETCH OF PROOF. The first identity follows from the fact that

$$
\widetilde{A}=\left(\begin{array}{cc}
0 & 0 \\
0 & \widetilde{S}
\end{array}\right)+\text { compact operator in } L^{2}(\Omega)^{q} .
$$

In the second identity, the inclusion $\subset$ is immediate; on the other hand, when $\mu$ is an eigenvalue for $\sigma^{0}(\widetilde{S})\left(x_{0}, \xi_{0}\right)$ with eigenvector $\theta, \widetilde{S}-\mu$ has the singular sequence (in a local coordinate system where $x_{0}=0$ )

$$
w_{k}(x)=k^{n / 2} v(k x) \exp \left(i\left\langle x, k^{2} \xi_{0}\right\rangle\right) \theta, \quad k \rightarrow \infty,
$$

where $v \in C_{0}^{\infty}\left(\mathbf{R}^{n}\right)$ with $v(0)=1$ and $\|v\|_{0}=1$. Finally, the last identity follows from the equation, valid for $\lambda \in \mathbf{C}$,

$$
\operatorname{det} \sigma^{0}(A-\lambda)=\operatorname{det} \sigma^{0}(I-\lambda \widetilde{S}) \operatorname{det} \sigma^{0}(A) .
$$

REMARK. ess sp $A$ is bounded if and only if $P$ (cf. (1.1)) is elliptic.

2.2. Furthermore, assume now that $A$ is strongly elliptic (i.e., $\sigma^{0}(A)+$ $\sigma^{0}(A)^{*}$ is positive definite on $\left.T^{*}(\bar{\Omega}) \backslash 0\right)$ and formally selfadjoint. Then, in particular, $P$ is strongly elliptic, so ess sp $A$ is bounded. Since $A$ is unbounded (and selfadjoint, lower bounded), it has a sequence of eigenvalues $\lambda_{j}^{+}(A)$ converging to $+\infty$ for $j \rightarrow \infty$. For large $\lambda$, the eigenvalue problem

$$
(P-\lambda) v+Q w=0, \quad Q^{*} v+(M-\lambda) w=0
$$

is equivalent with the nonlinear problem

$$
\left(P-Q(M-\lambda)^{-1} Q^{*}\right) v-\lambda v=0 .
$$

Here $P-Q(M-\lambda)^{-1} Q^{*}$ approaches $P$ as $\lambda$ becomes large, so (2.6) approaches an eigenvalue problem for $P$ in some sense. Indeed, we can show (see [5]): 
THEOREM 2.2. Let A be strongly elliptic and formally selfadjoint. The spectrum of $A$ on $]\|M\|,+\infty\left[\right.$ is a sequence of eigenvalues $\lambda_{1}^{+}(A) \leqslant$ $\lambda_{2}^{+}(A) \leqslant \cdots$ (repeated according to multiplicities) converging to $+\infty$ as follows: $\lambda_{j}^{+}(A) \sim \lambda_{j}(P) \sim c j^{2 m_{q^{\prime} / n}}$ for $j \rightarrow \infty$, where $c$ is a constant determined from $\sigma^{0}(P)$, and $a_{j} \sim b_{j}$ for $j \rightarrow \infty$ means $a_{j} / b_{j} \rightarrow 1$ for $j \rightarrow \infty$.

\section{The case of a manifold with boundary.}

3.1. Consider now the case where $\Gamma \neq \varnothing$. We may assume that $\bar{\Omega}$ is smoothly imbedded in an $n$-dimensional $C^{\infty}$ manifold $\Sigma$ without boundary, $A$ extended to an elliptic operator, also called $A$, on $\Sigma$. Let $\widetilde{A}$ be a parametrix of $A$ on $\Sigma$ (a properly supported $q \times q$-matrix pseudodifferential operator satisfying $\sigma(A \widetilde{A})=\sigma(\widetilde{A} A)=I)$.

Let $A_{B}$ be the $L^{2}$-realization of $A$ in $\Omega$ determined by (1.2):

$$
D\left(A_{B}\right)=\left\{u \in L^{2}(\Omega)^{q} \mid A u \in L^{2}(\Omega)^{q},(1.2) \text { holds }\right\} .
$$

It is well known that $A_{B}-\lambda$ is Fredholm as an operator from $\Pi_{t=1}^{q} H^{m+m_{t}}(\Omega)$ to $\Pi_{t=1}^{q} H^{m-m_{t}}(\Omega)$ (bounded operator!) if and only if (1.2) covers $A-\lambda$. For unbounded realizations, one may have the Fredholm property without covering (we have examples where the unbounded realization in $\Pi_{t=1}^{q} H^{m-m}(\Omega)$ falls into the uniformly nonelliptic class of Vainberg and Grusin [6] ). However, we have found for the $L^{2}$-realization:

THEOREM 3.1. Let $\omega=\{\lambda \mid A-\lambda$ is not elliptic on $\bar{\Omega}\}, \omega_{B}=$ $\{\lambda \mid \lambda \notin \omega$ but (1.2) does not cover $A-\lambda\}$. Then

$$
\text { ess sp } A_{B}=\omega \cup \omega_{B} \text {. }
$$

As the proof is technically much more involved than that of Theorem 2.1, only some ingredients will be mentioned: We use the theory of Boutet de Monvel [3] to construct a parametrix for $A_{B}$. It makes sense on $L^{2}(\Omega)$ thanks to the essential observation that $\widetilde{\mathbb{C}}^{01} \beta^{1} \widetilde{A}$ is a trace operator of class 0 (in the sense of [3]), so acts on $L^{2}(\Omega)$, in contrast to $\beta^{1} \widetilde{A}$. Another important point is that $\omega_{B}$ is contained in the point spectrum of the symbol of a certain singular Green operator of order 0 , associated with $A_{B}$-examples in [5] show that, in general, $\omega_{B} \neq \varnothing$.

3.2. For the case where $A$ is strongly elliptic and formally selfadjoint, we find for the selfadjoint, lower bounded realizations (which may be character- 
ized by use of [4] ) the analogous results to those in $\S 2.2$ : ess sp $A_{B}$ is bounded, and the sequence of eigenvalues going to $\infty$ behaves approximately like the sequence of eigenvalues for a corresponding selfadjoint realization of $P$.

REMARK. By similar techniques one may study the spectral theory for the "boundary value problems with potentials" considered in Baouendi-Geymonat [2] , and for certain boundary problems for pseudodifferential operators.

\section{BIBLIOGRAPHY}

1. S. Agmon, A. Douglis and L. Nirenberg, Estimates near the boundary for solutions of elliptic partial differential equations satisfying general boundary conditions. II, Comm. Pure Appl. Math. 17 (1964), 35-92. MR 28 \#5252.

2. M. S. Baouendi and G. Geymonat, Transposition des problèmes aux limites elliptiques, Symposia Math. 7 (1971), 421-441.

3. L. Boutet de Monvel, Boundary problems for pseudo-differential operators, Acta Math. 126 (1971), 11-51.

4. G. Grubb, Weakly semibounded boundary problems and sesquilinear forms, Ann. Inst. Fourier (Grenoble) 23 (1973), 145-194.

5. - Essential spectra of elliptic systems on compact manifolds, Spectral Analysis (C. I. M. E., 1973), Edizioni Cremonese, Roma, 1974, pp. 141-170.

6. B. R. Vaĭnberg and V. V. Grǔsin, Uniformly nonelliptic problems. II, Mat. Sb. 73 (115) (1967), 126-154 = Math. USSR Sb. 2 (1967), 111-134. MR 36 \#552.

7. F. Wolf, On the essential spectrum of partial differential boundary problems, Comm. Pure Appl. Math. 12 (1959), 221-228. MR 21 \#6472.

ISTITUTO MATEMATICO, POLITECNICO DI TORINO, TORINO, ITALY DENMARK

MATEMATISK INSTITUT, UNIVERSITETSPARKEN 5, 2100 COPENHAGEN, 